\title{
Lipid oxidation and perceived exertion level during exercise in obese: effect of the exercise form
}

\author{
Mohamed Ali Khanfir ${ }^{1}$, Hassen Ben Aouicha ${ }^{1}$, Liwa Masmoudi ${ }^{1}$, \\ Faten Ben Hmadou ${ }^{2}$ \\ ${ }^{1}$ Higher Institute of Sport and Physical Education Sfax (Tunisia) \\ ${ }^{2}$ Sectorial center of medicine and sports sciences Sfax (Tunisia)
}

\begin{abstract}
Regular exercise is one of the most used solutions to avoid obesity. In this study we compared the amounts of lipid oxidation and the level of perceived exertion in three physical exercises, one continuous and two intermittent in obese. Ten obese men (age $26.01 \pm 6.0$ years, weight: $104.2 \pm 19.4 \mathrm{~kg}$, BMI: $33.5 \pm 3.6 \mathrm{~kg} /$ $\mathrm{m}^{2}$ ) performed three 45 minutes exercises during which we measured energy expenditure and the level of perceived exertion. A continuous exercise whose intensity corresponds to the intensity of Fat max, an intermittent exercise which alternate four minutes at the intensity of Fat max $-10 \%$ and one minute at the fat max intensity $+10 \%$ (intermittent 1/4), and a second intermittent exercise which alternate two minutes at the intensity of Fat max $-10 \%$ and one minute at the Fat max intensity $+20 \%$ (intermittent 1/2). While the total energy expenditure during continuous exercise (321.6 Kcal) is higher than those of the intermittent 1/4 (268.1 $\mathrm{Kcal})$ and the intermittent 1/2 $(268.9 \mathrm{Kcal})$, the amounts of energy from oxidized fats in the three exercises are equivalent: 34,6 Kcal, 31,8 Kcal and 36,2 Kcal respectively for the three exercises. The perceived exertion measured by the Borg scale showed that intermittent exercises causes less fatigue in obese than the continuous exercise.
\end{abstract}

Keywords: Obese, exercise form, fat expenditure, perceived exertion

\section{Introduction}

Obesity has become the first non-infectious disease of history [1].The World Health Organization puts its prevention and its management as a priority in the field of nutritional pathology [2]. The obesity treatment is faced difficulties fit for any disease with a strong behavioral and social component [3] [4]. The management of the obese can combine several methods: diet, behavioral therapy, drug therapy, endoscopic and surgical treatment and physical exercise [5]. Currently, regular physical activity is part of most strategies for management of obesity [6]. The metabolic entrainment became a reality [7]. There is some consensus to promote physical activity in the fight against obesity [8]. The prescription, however, still seems a perfectible area. The intensity and duration of exercises for obese have been the object of several studies whereas studies that have dealt with the form of these exercise are rare [9]. With the emergence of the "Crossover Point" and "fat max" concepts, the determination of the optimal exercise intensity for fat oxidation is now individualized [10]. In fact, several studies emphasize the importance of the individualized intensity in physical activity programs [10] [11]. The duration of the proposed exercises for the obese varies from thirty to sixty minutes [12]. With obese, the question that is often debated now is no longer the intensity or the duration of the exercise, but the form of the exercise. Until now, the continuous exercise is the mostly recommended despite the interests of the intermittent exercise. Continuous and prolonged exercise is known for its ability to oxidize large amounts of lipids [13] [14]. Therefore, the rehabilitation programs offered to obese patients are often composed of continuous aerobic exercise [15] [16]. Moreover, relatively recent studies have shown the interest of intermittent exercise on the mobilization and oxidation of lipids [17] [18] [19]. In Obese patients, in addition to the intensity, duration and form of the exercise, the effectiveness of a training program depends largely on the level of patient's adherence. If obese patients adhere little to rehabilitation programs, the benefits are often disappointing. In overweight persons, the stress level of exercise is very important for the sustainability of a regular exercise. A low to moderate perception of stress exercise is essential [20]. The aim of any program in management of obese is to transform an inactive person to an active [15]. In this register, the intermittent exercise appears to be less monotonous and better perceived than continuous exercise. In this study we will compare the evolution of lipid oxidation and the level of stress generated by three physical exercises, one continuous and two intermittent, in obese subjects.

\section{Methods}

Ten obese men (age $26.01 \pm 6.0$ years, weight: $104.2 \pm 19.4 \mathrm{~kg}$, height: $1.75 \pm 0.088 \mathrm{~m}$, BMI: $33.5 \pm$ $3.6 \mathrm{k} / \mathrm{gm} 2)$ participated in the study. A doctor specializing in sports medicine center conducted a consultation 
for participants to verify that they are nonsmoking, free from diabetes, hypertension, and coronary heart disease, and they were not taking medications known to influence metabolic responses. Subjects were instructed not to engage in any strenuous exercise on the day preceding an experimental test, and they participated in 4 separate exercise sessions on cycle ergometer (Ergometrics 800, Ergoline ®, Blitz, Germany) spaced between 3 to 5 days from each other. A first session consisting in a progressive exercise for the determination of $\mathrm{VO}_{2 \max }$ and Fat max. The other three sessions consist of three 45 minutes exercises which simulate exercise training where we measure the oxidation of fats and carbohydrates while noting the level of perceived exertion. A continuous exercise whose intensity corresponds to the intensity of Fat max, an intermittent exercise which alternate four minutes at the intensity of Fat $\max -10 \%$ and one minute at the fat max intensity $+10 \%$ (intermittent $1 / 4$ ), and a second intermittent exercise which alternate two minutes at the intensity of Fat max $-10 \%$ and one minute at the Fat max intensity $+20 \%$ (intermittent $1 / 2$ ).

The order of the 45 minutes exercise was randomized and followed a counterbalanced format. Before participating in the study, all subjects were informed of the protocol and the objective of this study and gave their written consent.

\subsection{Testing protocol}

The progressive exercise used here was adapted from a previously described and validated protocol in obese [6]. The progressive exercise includes an initial warm-up period at $20 \%$ of the Maximum Predicted Power (MPP) for three minutes. After this warm-up phase, the test has four levels of six minutes long at 30, 40, 50 and $60 \%$ of the MPP respectively. Oxygen uptake $\left(\mathrm{VO}_{2}\right)$ and carbon dioxide production $\left(\mathrm{VCO}_{2}\right)$ were averaged over the last 2 min of each six minutes level. The six minutes' Levels are more suitable for obese patients than three minutes' levels. After the last step of six minutes, two to three short steps of one minute long have been made to meet the classical criteria of maximality of the test. The recovery phase includes two periods when monitoring respiratory and cardiac parameters is maintained: active recovery at $20 \%$ of the MPP for one minute and passive recovery during the next two minutes.

For the determination of fat max, breath-by-breath measurements were taken during exercise by using an open circuit gas analysis system (ZAN 600 WHOLE SYSTEM TB600/005) to measure $\mathrm{VO}_{2}$ and $\mathrm{VCO}_{2}$. From these values, we determine the respective shares of oxidation of carbohydrates and lipids by applying the theory of indirect calorimetry that uses the following formulas:

Carbohydrates $(\mathrm{mg} / \mathrm{min})=4,585 \mathrm{VCO}_{2}-3,2255 \mathrm{VO}_{2}$

Lipids $(\mathrm{mg} / \mathrm{min})=-1,7012 \mathrm{VCO}_{2}+1,6946 \mathrm{VO}_{2}$

In the 45 minutes exercises, measuring $\mathrm{VO}_{2}$ and $\mathrm{VCO}_{2}$ have determined the respective shares of carbohydrates and lipids in energy expenditure. The use of the Borg scale has determined the level of perceived exertion at the end of each exercise [21].

\subsection{Statistical analyze}

The statistical data analysis is performed on a microcomputer by using the software Statistica 6.1. The data are presented in the text and in the tables as means \pm standard deviations and in the figures as mean and standard errors. Shapiro Wilk test is applied to check the normality of distributions. The comparison between the three types of exercise is done by the application of an analysis of variance (ANOVA) with repeated measures. When a significant effect was observed, a post hoc analysis Scheffé test is conducted to compare the types of exercise in pairs. All statistics are considered significant for a probability threshold of 5\% (p<0.05).

\section{Results}

The lipid flow at Fat max measured during the progressive test is $133.8 \mathrm{mg} / \mathrm{min} \pm 33.4$ which complies with the results of F. Brandou et al. [22] and JF Brown et al. [15]. The Fat max of study subjects corresponds to $30.3 \pm 5.3 \%$ of $\mathrm{VO}_{2 \max }$ in accordance to the literature [23] [15] [24] [22]. Total energy expenditure during the three exercises of 45 minutes is reported in Fig 1. 


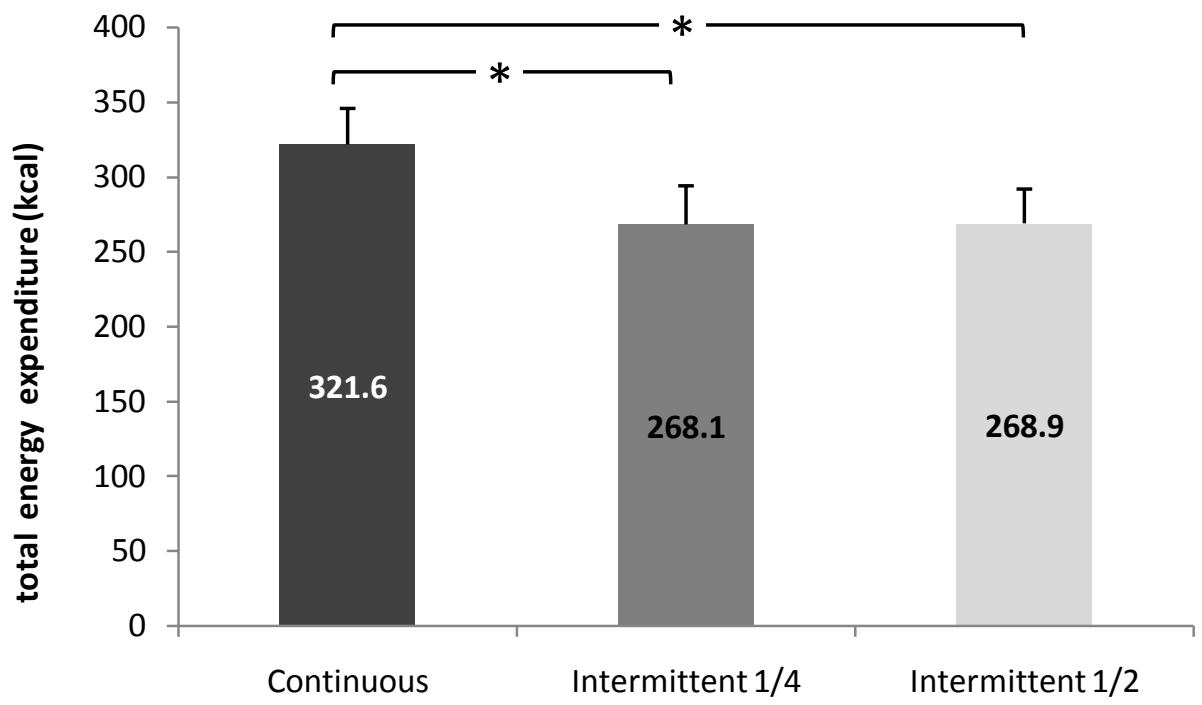

$1 / 2$ exercise

Fig 1: Total energy expenditure during continuous exercise, intermittent $1 / 4$ exercise and intermittent

*: significant difference $(\mathrm{p}<0.05)$

The variance analysis showed a significant effect between the three types of exercises: $\mathrm{F}(2,18)=$ 155.08. The post hoc test shows a significant difference between the continuous exercise and intermittent exercises $\mathrm{p}<0.001$.

The share of fats and carbohydrates in energy expenditure is reported in Fig 2.

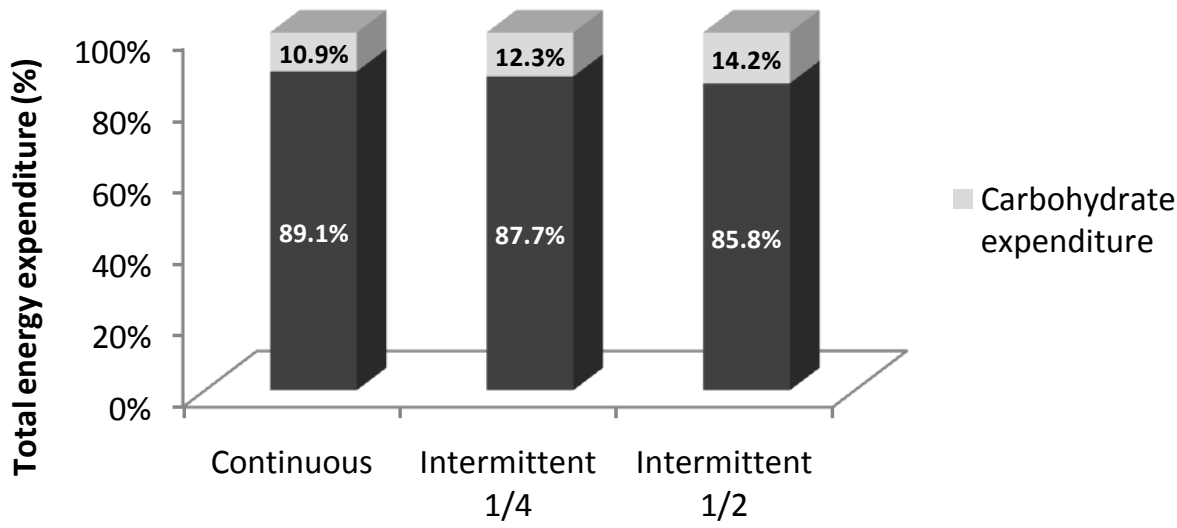

Fig 2: Relative energy expenditure during continuous exercise, intermittent $1 / 4$ exercise and intermittent $1 / 2$ exercise

The analysis of variance did not show a significant difference between the three types of exercises: $\mathrm{F}$ $(2,18)=0.854, \mathrm{p}=0.442$.

While the total energy expenditure during continuous exercise $(321.6 \mathrm{Kcal})$ is higher than those of the intermittent $1 / 4(268.1 \mathrm{Kcal})$ and the intermittent $1 / 2(268.9 \mathrm{Kcal})$, the percentage of fat expenditure during continuous exercise is lower than the percentage of fat expenditure during intermittent exercises.

The amounts of oxidized fats in the three exercises are equivalent. The variance analysis of amounts of burned fat during the three exercises showed no significant difference: $F(2,18)=0,321$, $\mathrm{p}=0,729$ Fig 3 . 


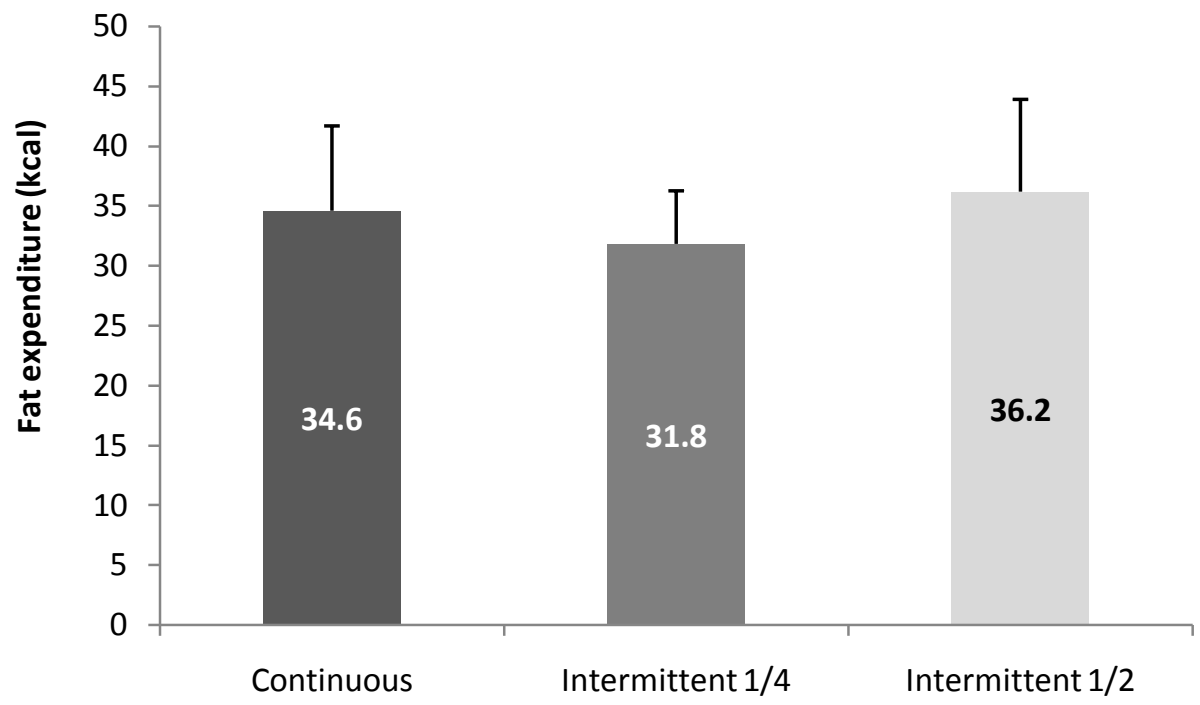

Fig 3: Energy from lipid expenditure during continuous exercise, intermittent $1 / 4$ exercise and intermittent $1 / 2$ exercise

The analysis of perceived exertion by the Borg scale during the three exercises of 45 minutes showed that intermittent exercises causes less fatigue in obese than the continuous exercise Fig 4 .

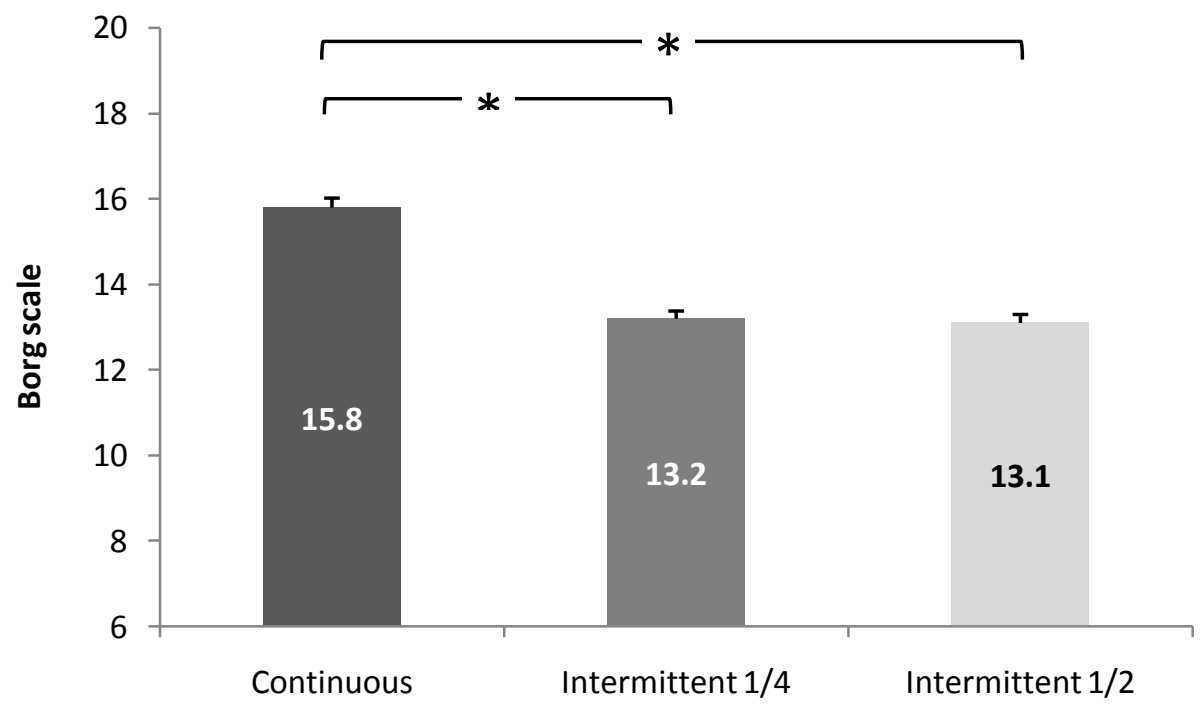

Fig 4: Effect of the exercise form on the perceived exertion during continuous exercise, intermittent 1/4 exercise and intermittent $1 / 2$ exercise

The analysis of variance showed a significant effect between the three types of exercises with $F(2,18)$ $=65.23$. The post hoc test displayed a significant difference between the exercise in its continuous and intermittent form at $\mathrm{p}<0.001$

\section{Discussion}

The best form of exercise for fat utilization is subject of controversy. Exercises usually offered during retraining programs for obese are continuous exercises [14] [10] [8] [16] while several studies have indicated that intermittent exercise may increase fat oxidation compared to continuous exercise [17] [18]. Besides, the proposed activities to obese should ensure an optimal level of membership. A perception of low to moderate stress exercise is essential [20].

Based on previous findings, we saw interesting to conduct a study of the oxidation of lipids in obese during different types of exercise to determine the most effective and better perceived exercise by this population. 
On total energy expenditure, we found that continuous exercise allows more total expenditure (321.6 kcal) than the intermittent exercise $1 / 2(268.1 \mathrm{Kcal})$ and intermittent exercise $1 / 4(268.9 \mathrm{Kcal})$. With daily practice, these amounts of energy expenditure for the three types of exercise exceed the weekly standard threshold of $1500 \mathrm{Kcal}$ recommended by GM Gakicic et al [13] JM Oppert and [25].

Although the total amount of energy expended during continuous exercise is higher than the amount of energy expended in intermittent exercises, the relative amount of energy from fat in the continuous exercise $(10.9 \%)$ is lower than the intermittent exercise $1 / 4(12.3 \%)$ and the intermittent exercise $1 / 2(14.2 \%)$.

These measures suggest that intermittent exercise is more selective in lipid oxidation than continuous exercise. This idea is confirmed by Tremblay et al [26] who have compared a continuous exercise program of 20 weeks to intermittent exercise program of 15 weeks. In this study, the energy cost of intermittent exercise was very lower than the energy cost of the continuous exercise but the impact of intermittent exercise on the reduction of subcutaneous fat was significantly higher. In another study, Billat [28] showed that intermittent exercise increased oxidative capacity of muscle fibers type II better than continuous exercise. Well, Essen et al [28] showed that an hour of intermittent exercise around 50\% of $\mathrm{VO}_{2 \text { max }}$, lipids were more used than glycogen when compared with continuous exercise at $50 \%$ of $\mathrm{V}_{\mathrm{O} 2 \max }$.

The continuous exercise has long been used by obese subjects [29] [16] [30] since it is more costly in terms of energy. The results we have identified show that the intermittent exercise is less expensive in energy expenditure but more selective in the oxidation of lipids. The intermittent exercise affinity to lipid oxidation will probably guide the choice of several authors to this type of exercise in oxidation fat program [18].

Up to now, studies which suggest intermittent exercise are very rare. To our knowledge, we can expose two works. The study of Gail Trapp and al [17] consisted of short sprints (8 seconds sprint, 12 seconds recovery) and long sprints (24 seconds sprint, 36 seconds recovery) for 20 minutes. And the study of B.J. Coquart et al. [18] which consisted of 32 minutes of intermittent exercise on a cycle ergometer. During these exercises, subjects alternated two minutes of work at $120 \%$ of the aerobic threshold and two minutes of active recovery at $80 \%$ of the aerobic threshold. In these studies, exercise intensities had now relationships with the lipid oxidation intensity. As recognized by F. Brandou, If we seek to optimize lipid expenditure, the exercise intensity should be individualized at lipids maximum oxidation point (Lipox max) if exercise is continuous and around this point if exercise is intermittent [31].

The effectiveness of a training program in obese is not limited to the amount of fat oxidized during exercise but is measured by the sustainability of its application. It is encouraging inactive and sedentary subjects to become moderately active on a regular basis. In other words, we try to integrate physical activity into the welfare registry and improving the health of humans [25] [3].

In our study, the intermittent exercise is better perceived by obese subjects. These subjects consider continuous exercise as difficult whereas they consider intermittent exercises to be just somewhat difficult. This result is consistent with the result suggested by B. J. Coquart which advocates that the intermittent exercise is the best perceived form by obese [18]. Compared with continuous exercise the intermittent exercise can oxidize the same amounts of lipids with less tiredness and less energy expenditure fatigue and monotony. These results should encourage the intermittent exercise prescription instead of continuous exercise in the treatment of obesity.

\section{Conclusion}

Through this work, definitely we have determined the most appropriate exercise form for lipid oxidation in obese subjects. However, we can think to improve the effectiveness of this exercise. The duration of this exercise can probably be extended, to increase energy expenditure, views the reduction of fatigue recorded in this form of intermittent exercise.

\section{References}

[1] F. Samguigol, Obesity: A Chronic multifactorial disease, Physiotherapy, review, Volume 8 issue, in April, 2008, pages.14-16.

[2] C. Emery, J. Dinet, A. Lafuma, C. Sermet, B. Khoshnood, F. Fagnani, Evaluation of stroke associated with obesity in France, Metabolic disease medicine, Volume 1 issue 2 June 2007, Page 28-34.

[3] C.A. Dour, T. M. Horacek, S. M. Schembre, B. Lohse, S. Hoerr, K. Kattelmann, A. A. White, S. Shoff, B. Phillips, G. Greene Process Evaluation of Project WebHealth: A. Nondieting Web-based Intervention for Obesity Prevention in College Students. $J$ Nutr Educ Behav. 2013, Feb 11.

[4] M. C. Kegler, D. W. Swan, I. Alcantara, L. Feldman, K. Glanz, The Influence of Rural Home and Neighborhood Environments on Healthy Eating, Physical Activity, and Weight.Prev Sci. 2013, Feb 14.

[5] M. MERROUCHE, B. Coffin. Obesity treatment, indication and method of Endoscopic and surgical treatment, EMC-Hepatho Gastroenterology, Volume 2, issue 3 July 2005, page 189-200.

[6] C. Grondard, F. Charbonnier, J. P. Gasc, P. P. Vidal, C. L. Gallien, C. Canon, Biological responses to training: data cineradiography. Science \& Sports vol. 20 Issue 4 August, 2005. p. 232-234.

[7] J. K. Zakrzewski, K. Tolfrey, Comparison of fat oxidation over a range of intensities during treadmill and cycling exercise in children, Eur J Appl Physiol, 2012 Jan;112(1):163-71. 
[8] D. R. Bensimhon, W. E. Kraus, and M. P. Donahue, “Obesity and physical activity: a review,"American Heart Journal, vol. 151, no. 3, pp. 598-603, 2006.

[9] B. J. Coquart and al. Chronic Effects of intermittent exercises on energy intake in obese women: a preliminary study, Journal Obesity, Volume 4, Number 1, March 2009, Pages 49-53.

[10] JF Brun, John E, E Ghanassia, Flavier S, Mercier J. Retraining metabolic diseases targeted individually by calorimetry effort. Annals of Physical Medicine and Rehabilitation 2007; 50 (6):520-527

[11] N. A. Crisp, K. J. Guelfi, M. K. Licari, R. Braham, P. A. Fournier, Does exercise duration affect Fatmax in overweight boys? Eur J Appl Physiol, 2012 Jul; 112(7),2557-64.

[12] P. Duché, Physical activity and infantile obesity: Tracking, prevention and treatment, Science \& Sports 23 (2008) $278-282$.

[13] J. M. Jakicic, Physical activity considerations for the treatment and prevention of obesity, Am J Clin Nutr, 2005, 82(suppl 1), 226S$229 \mathrm{~S}$.

[14] M. C. Venables, A. E. Jeukendrup, Endurance training and obesity: effect on substrate metabolism and insulin sensitivity, Med Sci Sports Exerc, 2008 Mar;40(3):495-502.

[15] I. Glisezinski, Lipid mobilization from adipose tissue during exercise, Science \& Sports vol. 22 Issue 6 December, 2007, p. 280285.

[16] R. C. Browning, M. M. Reynolds, W. J. Board, K. A. Walters, Obesity does not impair walking economy across a range of speeds and grades, J Appl Physiol, 2013 Feb 14.

[17] E. G. Trapp, D. J. Chisholm, S. H. Boutcher, Metabolic response of trained and untrained females during high intensity intermittent cycle exercise, Am J Physiol Regul Integr Comp Physiol, 2007.

[18] J. B. J. Coquart, C. Lemaire, C. Douillard, M. Garcin, Effects of an intermittent walking program on mass and body composition in obese women, Journal of Endocrinology Volume 69, Issue 3, June 2008, Pages 227-230.

[19] J. R. Lacour, Lipid metabolism and exercise, The practitioner Review 2001, vol. 51, No. JUN, NS, p. 36-41.

[20] S. Lazzer, M. Vermorel, C. Montaurier, Changes in adipocyte hormones and lipid oxidation associated with weight loss and regain in severely obese adolescents, Int. J. Obes, (Lond.),2005;29:1184-1191.

[21] G. BORG, Borg's perceived exertion and pain scales, Human Kinetics, 1998, p 103.

[22] F. Brandou, M. Dumortier, P. Garandeau, J. Mercier, J. F. Brun, Effects of two-month rehabilitation program on substrate utilization during exercise in obese adolescents, Diabetes and Metabolism, (2003). 29, 20-27.

[23] E. Garrigue, I. Glisezinski, I. Harant, C. Moro, F. Pillard, F. Cramps, , D. Rivière, Lipid metabolism and muscular exercise in obese subjects. Science \& Sports vol. 21 issue 2 April, 2006. p. 68-73.

[24] A. Perez-Martin, M. Dumortier, E. Raynaud, J. F. Brun, C. Fedou, J. Bringer, Balance of substrate oxidation during submaximal exercise in lean and obese people, Diabetes Metab 2001; 27: 466-74.

[25] J. M. Oppert, P. Dalarun. Physical activity and obesity treatment, Medicine Obesity, BASDEVANT A GREAT GUY-B (eds), Medicine-Flammarion Sciences, Paris, 2004: 222-227.

[26] A. Tremblay, J. A. Simoneau and C. Bouchard, Impact of exercise intensity on body fatness and skeletal muscle metabolism, Metabolism, 1994, 43:814-8.

[27] V. Bïllat, B. Flechet, B. Petit, Interval training effects on aerobic performance and overtraining markers, Med Sci Sports Exerc, $1999 ; 31: 156-63$.

[28] B. Essén, Glycogen depletion of different fiber types in human skeletal muscle during intermittent and continuous exercise, Acta Physiologica Scandinavica, Volume 103, Issue 4, August, 1978, p 446-455.

[29] E. John, Grubka, Mr. Karafiat, S. Flavier, C. Fédou and J. Mercier, Effects of endurance training targeted by calorimetry effort among type 2 diabetes, Ann. Endocrinol, (Paris) 67 (2006), p. 462.

[30] M. J. Ekman, M. Klintenberg, U. Björck, F. Norström, M. Ridderstråle, 6-minute walk test before and after a weight reduction program in obese subjects, Obesity (Silver Spring). 2012 Oct 3.

[31] F. Brandou, A.M. Savy-Pacaux, J. Marie, M. Bauloz, I. Maret-Fleuret and S. Borrocoso, Impact of high and low-intensity targeted exercise training on substrate utilization in obese boys submitted to a hypocaloric diet, Diabetes Metab, (2005), p 327-335. 